\title{
Problemática en torno a Rom 13,1-7: El cristiano y la autoridad civil
}

\section{PROBLEMAS DE CONTEXTO Y TRADICIÓN}

Dentro de la discusión general que circunda el fragmento, el problema de su relación con el contexto inmediato es uno de los más enconados, pues los exégetas han llegado a las opiniones más dispares y contradictorias. Desde considerarlo una interpolación, como Schmitlals ' , a pensar que se sigue con cierta normalidad de su contexto, como Wilckens ${ }^{2}$.

En este orden - o desorden - de cosas, la opinión más comúnmente admitida era la de Käsemann, que dice: «Nuestro fragmento es un bloque independiente, que con mayor razón debido a su singular finalidad, puede ser calificado como un cuerpo ajeno en la parénesis paulina... Debe ser interpretado primeramente a partir de si mismo» ${ }^{3}$.

Wilckens resume los argumentos que propone esta tendencia para sostener su opinión ${ }^{4}$ : a) El fragmento se diferencia estilísticamente de lo precedente en que introduce un lenguaje jurídico y de diatriba y rompe con el lenguaje en segunda persona (propio de la parénesis), al que retorna en el v. 7. b). E leitmotiv del c. 12 es el amor entre sí y a los enemigos, mientras que en 13,1-7 falta la alusión al amor. Todo aparece más bien en un contexto jurídico. No se manda amar a las autoridades. c) En el v. 7 se desvía Pablo del tema del amor. En cambio, en 13,8 lo introduce bruscamente, empalmando así con 12,21. Nuestro fragmento sería un excursus cerrado en sí mismo. d) Lo cerrado del

1. W. SCHMITHALS, «Der Römerbrief als historisches Problem»: StNT, 9 (1975) 185187. Citado por J. FRIEDRICH-W. POEHLMANN-P. STUHLMACHER, «Zur historischen Situation und Intention von Röm 13,1-7): Zeitschrift für Theologie und Kirche, 73 (1976) 131.

2. U. WILCKENS, «Römer 13,1-7»: Rechtfertigung als Freiheit. Paulusstudien, Düsseldorf, 1974, 216.

3. E. KAESEMANN, An die Römer, Tübingen 1974, 340. Ver también del mismo autor: Ensayos exegéticos, Salamanca 1978, 32.

4. U. WILCKENS, o. c., 205-207. 
texto se explica por su procedencia de un topos tradicional, al que también pertenecen 1Pe 2,13-17; Tit 3,1s. y $1 \mathrm{Tm} 2,1 \mathrm{~s}$. Esto se ve por el conjunto de diferencias y convergencias de los textos entre sí y con Rom 13,1-7. e) Por la historia de las formas se puede ver que la tradición parenética se caracteriza por la falta de ligazón en los motivos, según un esquema de análisis interno. Están unidos más bien desde fuera. Käsemann dice que «la construcción de la parénesis paulina se forma en sus detalles por coordinación, no por subordinación ni deducción, por asociación y no por lógica» ${ }^{5}$.

A estos argumentos hay que hacerles importantes objeciones.

\section{Lenguaje y tradición}

a) En cuanto a los textos paralelos en Rom 13,1-7: $1 \mathrm{Pe} 2,13-17 ; 1 \mathrm{Tm}$ 2,1 s y Tit $3,1 \mathrm{~s}$, Wilckens da los siguientes argumentos para el entendimiento de nuestro texto a partir de sí mismo ${ }^{6}$ : El pensamiento de la institución de las autoridades por Dios se encuentra sólo en Rom 13,1-7. Rom 13, 1a está formulado en general, los otros textos son exhortaciones concretas. No hay ningún paralelo a Rom 13,5. Sólo en nuestro texto se habla de pago de los impuestos.

A esto añaden Friedrich-Pöhlmann-Stuhlmacher: ${ }^{7}$. Las palabras tò agathón, diákonos theoû, orgé, syneldesis, leitourgol theoû, apodidomi, son típicas paulinas y se hallan sólo aquí. Términos como diatagé, máchaira, phoréo, phóros y télos, que tienen un significado muy concreto, no aparecen en los otros textos.

Con esto y con las notables concordancias que también se encuentran ${ }^{8}$, se concluye: «Los estrechos contactos entre 1Pe 2,13-17; 1Tm 1,1s; Tit 3, 1s y Rom 13,1-7 no se explican por un uso literal directo. Todos los textos parecidos dependen más bien de la primitiva parénesis cristiana sobre el comportamiento recto en el mundo políticon ${ }^{9}$.

b) Por lo que respecta a las expresiones utilizadas por Pablo, Käsemann ${ }^{10}$, siguiendo a A. Strobel, dice que pertenecen al lenguaje de la administración helenística. Así, por ejemplo: exouslai hyperechoúsai y árchontes serían los innumerables funcionarios de la autoridad pública helenística; el épainos es el escrito oficial de alabanza, acostumbrado en Roma, dado a los ciudadanos ejemplares por sus relaciones con el César o por su

5. E. KAESEMANN, Ensayos exegéticos, 32.

6. U. WILCKENS, o. c., 211 ss.

7. FRIEDRICH-POEHLMANN-STUHLMACHER, a. c., 134-135.

8. U. WILCKENS, o. c., 213-214.

9. FRIEDRICH-POEHLMANN-STUHLMACHER, a. c., 135. U. WILCKENS, o. c., 211. E. DAESEMANN, An die Römer, 339.

10. E. KAESEMANN, An die Römer, 341-342. 
conducta; ékdikos eis orgén, el abogado para el juicio o también el «vengadorn; leitourgol, los empleados en las finanzas; diatagé también pertenecería a este lenguaje, haría relación a un sistema de orden concreto.

Sobre esta base, Wilckens ${ }^{11}$ y Friedrich-Pöhlmann-Stuhlmacher ${ }^{12}$ hacen una corrección. Objetan que no todo se explica por este lenguaje de la administración pública. Friedrich-Pöhlmann-Stuhlmacher, con un detallado estudio de las palabras diatagé y máchaira, llegan a la conclusión de que Pablo ha escogido sus palabras mucho mejor de lo que a primera vista parece. Estas dos palabras, en concreto, no tenían habitualmente el significado que supohen los trabajos antes citados, sino que se aplican más bien a relaciones privadas, algunas de ellas también jurídicas, pero con un matiz más general. Con estos autores podemos decir: «Estos ejemplos podrían bastarnos para mostrar que, por un lado, en el trillado camino de Strobel, Delling y van Unnik ya se profundizó fundamentalmente en la comprensión de Rom 13,1-7, y, por otra parte, queda sin embargo por hacer una amplia investigación que describa el comportamiento del habitante griegoparlante del Imperio de cara a las autoridades y los deberes para con ellas" ${ }^{13}$.

c) Esto se explica también por la utilización que Pablo hace de las tradiciones sinagogales y veterotestamentarias. Tanto Wilckens ${ }^{14}$ como FriedrichPöhlmann-Stuhlmacher ${ }^{15}$ ofrecen una recopilación del material presentado en los trabajos de P. Billerbecks, O. Eck y W. Schrage. Ideas como las de que la autoridad política ha sido instituida por Dios, la intimación a la obediencia a estas autoridades, el temor a los representantes del poder, la designación de las autoridades como servidores de Dios y el darles honra, se sorprenden en la literatura profética, sapiencial y la primitiva literatura judía. Por lo que se descubre la dependencia de contenido que hay entre Rom 13,1-7 y esta serie de textos.

\section{El contexto}

a) La opinión que considera el fragmento un cuerpo extraño (Käsemann) y la que incluso lo tiene por interpolación (Schmithals) alegan que se sale del contexto del «amor» en que está todo el c. 12. Sería vuelto a tomar en 13,8 mediante el medeni medén, que enlaza con el medeni de 12,21 y que se repite cinco veces a lo largo de 12,16-21. La unión mediante tàs opheilás $(13,7)$ y ophellete $(13,8)$ sería un mero nexo artístico.

Para Wilckens ${ }^{16}$ y Friedrich-Pölmann-Stuhlmacher ${ }^{17}$ esto resulta proble-

\footnotetext{
11. U. WILCKENS, o. c., 214-215.

12. FRIEDRICH-POEHLMANN-STUHLMACHER, a. c., 136-144.

13. Id., 144-146.

14. U. WILCKENS, o. c., 223-226.

15. FRIEDRICH-POEHLMANN-STUHLMACHER, a. c., 145-146.

16. U. WILCKENS, o. c., 207-209.

17. FRIEDRICH-POEHLMANN-STUHLMACHER, a. c., 148-153.
} 
mático. Siguiendo la estructuración que estos últimos autores proponen del texto, hay que decir:

Al programa de 12,1-2 sigue 12,3-16a, que se refiere a la vida común de los cristianos. Se distinguen en esta parte dos pequeñas secciones: $12,6-8$ que es una enumeración de carismas bajo el tema del métron písteos $(12,3 \mathrm{~b})$, y 12,9-13 que es un catálogo de virtudes bajo el motivo del agápe (sólo aquí es central este motivo, contra la opinión anterior). Después viene 12,16b-13,7, dominada por el motivo de vivir en paz con todos los hombres, no sólo con los cristianos, y bajo la exhortación, también presente en el anterior fragmento, de hacer en todo la voluntad de Dios. Vivir en paz con los enemigos significa no devolver mal por mal y dejar sitio al juicio de Dios. Vivir en paz con las autoridades significa someterse a ellas, pues son servidoras de Dios. $Y$ esto, precisamente, porque se trata de hacer el bien y no el mal, pero según el criterio cristiano. Por fin, 13,8-10 sería una recopilación de los motivos anteriores y la unión de todos ellos. Enlaza con lo inmediatamente anterior mediante el ophélete. Tò allélous agapán (13,8) vuelve a tomar 12,5.10.16, y el agapán tòn héteron $(13,8)$ el amor a los que no son de la comunidad.

Se desechan, pues, el agápe como leitmotiv. Se trata de que los cristianos hagan la voluntad de Dios en todos los aspectos de su vida, que hagan el bien y no el mal. Así, quedan unidos 12,2.9.17.21; 13,3b.4. La autoridad es vista en este último lugar precisamente bajo el aspecto de sancionadora de las buenas y las malas acciones. Palabras que unen son también ekdíkesis $(12,9)$ ékdikos $(13,4)$, timé $(12,10$ y 13,7$)$. El motivo del juicio de Dios es también común en 12,19 y $13,4 b .5$, así como la expresión semejante kakòn poiein $(13,4)$ kakòn ouk ergátsesthai $(13,10)$.

b) De esta forma se sale también al paso a la proposición alegada desde la historia de las formas como explicación para la no ligazón del fragmento con el contexto inmediato ${ }^{18}$ : que la parénesis paulina se hacía por asociación y no por deducción. Responde Wilckens: «En cuanto al argumento de la no ligazón de las perícopas parenéticas propuesto por la historia de las formas, puede considerarse acertado; sin embargo no debe desconocerse que en la yuxtaposición de los conjuntos parenéticos hay también claramente una sobreordenación de motivos por su contenido" ${ }^{19}$.

c) Hay, no obstante. otras objeciones referentes a lo incoherente que resulta poner un ejemplo concreto en un marco de exhortaciones generales. Esta opinión quedará superada claramente en el siguiente apartado, donde se estudiará el motivo actual de la exhortación. Pero, aun antes de eso: «No se necesita recurrir a la situación de los cristianos romanos para corregir esta

18. Ver notas 4 y 5.

19. U. WILCKENS, o. c., 215. 
impresión. Pablo da argumentaciones en 12,19 así como en 13,9 y presenta también un ejemplo en 12,4 , sin dañar por ello la unidad de su parénesis» ${ }^{20}$.

\section{EL MOTIVO ACTUAL DEL FRAGMENTO}

A pesar de ver la clara relación de nuestro fragmento con el contexto, hay algo que sigue chocando: la taxativa insistencia de Pablo en el sometimiento concreto al pago de los tributos e impuestos. Aun entendido como ejemplo, no se explica su concretez tan vívida.

Wilckens y otros han visto en esta alusión un argumentum e silentio para la procedencia de este motivo del logion de Jesús que aparece en Mc 12,13-17 par ${ }^{21}$. Friedrich-Pöhlmann-Stuhlmacher dicen que con las dos únicas palabras iguales en ambos textos - apódote y phóros - es «teológicamente muy osado hacer provenir nuestro pasaje de la relación Jesús-Pablo» ${ }^{22}$. Además, sigue sin plantearse un motivo histórico concreto por el que lógicamente hemos de preguntar ante una exhortación tan radical y concreta. De hecho, casi todos los autores han tenido que dar una razón de este tipo, aunque resulta insuficiente.

Käsemann ha dicho que "Pablo se dirige contra una actitud que es indiferente o incluso desprecia a las autoridades políticas, en la conciencia de ser habitantes de la ciudad celestial» ${ }^{23}$. Se trataría, pues, de un entusiasmo desenfrenado del tipo del de Corinto. K. Haacker pondría la razón en la intención que Pablo tenía de erradicar las tendencias antirromanas y zelotas de la comunidad ${ }^{24}$. Wilckens rechaza ambos motivos', diciendo que este entusiasmo no parece ser un fenómeno romano ${ }^{25}$, y que de las tendencias zelotas no sabemos históricamente nada ${ }^{26}$. Según él, Pablo, enterado por Prisca y Aquila, entre otros, de la situación romana, y contrariado por sus experiencias en Corinto, querría prevenir a la comunidad de una posible irritación contra Roma.

Todos estos motivos, sin embargo, no se salen de la esfera de la subjetividad de Pablo, y no es extraño que Wilckens diga: «E. Käsemann ha postulado con razón que la particular finalidad de Rom 13,1ss ha de explicarse desde el contexto de la teología paulina» ${ }^{27}$. No puede negarse que esto es necesario y posiblemente lo que más diga a la hora de hacer una reflexión teológica

20. FRIEDRICH-POEHLMANN-STUHLMACHER, в. c., 153.

21. U. WILCKENS, o. c., 226.

22. FRIEDRICH-POEHLMANN-STUHLMACHER, a. c., 156.

23. E. KAESEMANN, An die Römer, 338. Ensayos exegéticos, 42.

24. FRIEDRICH-POEHLMANN-STUHLMACHER, o. c., 132-133.

25. U. WILCKENS, o. c., 229.

26. Id., 228.

27. Id., 229. 
sobre el texto. Pero aquí se plantea la posibilidad de llegar a un dato objetivo que legitime la suposición de un motivo concreto histórico.

Se acepta, como principio, el dato de que Pablo estaba informado del estado de la comunidad. A partir de aquí son Friedrich-PöhlmannStuhlmacher ${ }^{28}$ quienes, siguiendo la iniciativa de 0 . Michel de investigar en los Annales de Tácito, han hallado en Ann. 13,50s. un informe interesante y clave: «Tácito informa aquí que el pueblo romano se decidió por fin en el año 58 d.C. a protestar ante el César por la rapiña de los recaudadores estatales de impuestos y tributos; el César consideró por algún tiempo después de esta protesta suprimir los impuestos en general, pero sus consejeros lo disuadieron de tomar esta decisión, inafrontable para las finanzas del Estado; por ello solamente reformó el aparato de impuestos y tributos» ${ }^{29}$. Con este informe podemos constatar que por el tiempo de la redacción de la carta a los romanos esta reforma todavía no se había realizado. Por tanto, las exhortaciones de Pablo tenían un significado muy concreto para la comunidad de Roma, que sufría el acoso de los recaudadores. Aun en estas circunstancias, Pablo exhorta a la obediencia y al cumplimiento.

Veremos en el siguiente apartado cuál es la razón profunda de todo ello. Pero, por ahora, es importante saber que la parénesis, como lo demuestra este ejemplo, puede tener en muchos casos motivos bastante más concretos de lo que se supone. No se asienta sobre hipótesis, sino que va dirigida a una situación real, lo que no obsta para que la fundamentación haya de ser aclarada por la teología paulina y por su experiencia de la vivencia cristiana en el mundo.

III. CONSECUENCIAS

\section{Lo que Pablo dice y lo que no dice}

Ya desde el siglo I, la Iglesia ha venido interpretando este texto de acuerdo con las circunstancias en que tenía que desarrollar su existencia en lo que afecta a sus relaciones con el poder político. Se han dado todo tipo de interpretaciones, la mayoría de ellas incluyendo una determinada doctrina o metafísica del Estado. Ha habido también demonizaciones y glorificaciones de la autoridad que se basaban en este texto. Pero todos los autores en que me baso coinciden en que en este fragmento no hay doctrina, ni metafísica ni demonización del Estado ${ }^{30}$.

Todos los argumentos tradicionales que Pablo aduce para exigir la obe-

28. FRIEDRICH-POEHLMANN-STUHLMACHER, a. c., 156-159.

29. /d., 157.

30. E. KAESEMANN, Ensayos exegéticos, 33-40. U. WILCKENS, o. c., 230-245. FRIEDRICH-POEHLMANN-STUHLMACHER, a. c., 160-166. 
diencia concreta a las autoridades no tienen nada que ver con la argumentación metafísica de nuestro tiempo. Hay que decir con Bornkamm: «Pablo dice todo esto sin hacer la menor alusión a la eventualidad de que la autoridad (pagana) pueda abusar de su poder, y crear así una situación que obligue al cristiano a obedecer a Dios antes que a los hombres (Hech 5,29 ), aparte de que este problema es conocido mucho antes del cristianismo" ${ }^{31}$. Las afirmaciones de Rom 13,1-4.6, que Schlier califica de «audaces formulaciones paulinas» 32, no afectan al juicio que podemos emitir sobre los representantes de la autoridad. Estarían en la línea de Jn 19,11: «No tendrías potestad alguna sobre mí si no te hubiera sido dada de lo alto». Aunque la opinión tradicional atribuía a Dios la creación de un mundo en el que todo estaba ordenado según un esquema de autoridades y súbditos, no quiere esto decir que el gobernante tuviera asegurado el obrar con justicia. Sería algo semejante a lo que significa en boca de los profetas la afirmación de la elección de Israel por Dios: no es únicamente un privilegio, sino también una exigencia. De igual forma, decir, con una mentalidad hebrea, que las autoridades han sido instituidas por Dios, es ambivalente. Se reconoce su puesto, que, debido a la época histórica, es atribuido a la intervención de Dios, pero es precisamente eso lo que ofrece el apoyo para una crítica profunda.

En cualquier caso, no es esto lo que aquí se plantea. Lo que Pablo quiere decir tiene mucha similitud de fondo con la exhortación -o más bien mandato - de 1 Cor 11,2 ss $^{33}$. Nadie duda que Pablo asume aquí las perspectivas conservadoras, incluso de aquel tiempo. Un estudio del texto nos hace comprobar que ni siquiera al mismo Pablo le convencen sus razones. Pero lo que el Apóstol persigue es precisamente poner en claro en qué consiste la libertad cristiana, en lo cual va en juego ni más ni menos que la esencia del ser cristiano. Ser cristiano en el mundo no consiste en liberarse de unas obligaciones, aunque sean injustas, sino en poner mi libertad a prueba mediante el servicio basado en la cruz de Jesús. La existencia cristiana es una tapeinophrosýne ${ }^{34}$. Pablo veía peligrar en Corinto la nueva dimensión que incorpora a la vida concreta la conversión cristiana, y no una simple costumbre de la que él estaba más liberado que nadie. Y esto era también lo que se podía estar conmoviendo en Roma. Podía desencadenarse una rebelión en la que nada había de cristianismo, que es esencialmente servicio. Por ello, lo fundamental de este texto debe permanecer, aunque la autoridad llegue a convertirse en un verdadero anticristo, como será experimentado más tarde, según aparece en el capítulo 13 del Apocalipsis ${ }^{36}$.

31. G. BORNKAMM, Paul, Apótre de Jésus-Christ, Genève 1971, 289.

32. H. SCHLIER, Der Römerbrief, Freiburg im Breisgau 1977, 392.

33. E. KAESEMANN, Ensayos exegéticos, 43-45.

34. Id., 31, 46.

35. G. BORNKAMM, o. c., 291. 
Conviene fijarse especialmente en el v. 5 de nuestro fragmento. Con Friedrich-Pöhlmann-Stuhlmacher opino que: "Si hay que buscarlo en algún sitio, es aquí donde recae el verdadero acento paulino de nuestro texto" ${ }^{36}$. Con la syneídesis introduce Pablo la instancia más crítica del cristiano ${ }^{37}$. Si hay gente que obedece por miedo o por simple lealtad cívica, el cristiano se somete dià tèn syneidesin. Es ella la que posibilita el auténtico martirio y el criterio para determinar cuándo ya no es posible la obediencia. Morir por un simple anhelo revolucionario no sería un martirio. "Es aquí donde radica el fundamento de toda esa variedad en la praxis que nos presentan los Hechos de los apóstoles: se va de la sumisión voluntaria al martirio, de la aceptación silenciosa de los malos tratos a la apelación ante la jurisdicción del César y a la afirmación de que hay que obedecer a Dios antes que a los hombres» ${ }^{38}$. En estos versículos vemos el anuncio del «acontecimiento de la salvación... que hace derrumbarse los límites del simple civismo" ${ }^{39}$. No se trata de debilidad, sino de la fuerza más radical. Algo de esto es lo que en nuestro siglo ha llevado a la práctica Gandhi con su doctrina de la resistencia pasiva.

Lo que hay en el fondo es la necesidad de vivir encarnado y, por tanto, limitado, y amar esa limitación - que puede exigir renuncias imprevisiblescomo única forma de superarla. Detrás de las expresiones de Pablo podíamos suponer - con mala idea - una ironía: «No tengáis prisa por rebelaros. Llegará el tiempo en que la misma autoridad que ahora os alaba por vuestro sometimiento carismático, también por él os castigará. Será entonces la autoridad quien se rebele contra vosotros, y no podréis hacer nada contra ello».

\section{El cristiano y la política}

Muchos cristianos han tratado de buscar en este texto una respuesta a su postura política. Pero, ya de entrada y por todo lo que antes se ha dicho, tenemos que ver la imposibilidad de tal planteamiento. Pablo presenta las autoridades como una cosa fáctica. Para él, pensar que el ciudadano - y menos el esclavo - en una sociedad a mil leguas de la democracia, podía participar en la formación del gobierno, era completamente imposible. Pablo no trata de dar una respuesta sociológico-política, sino de presentar el componente cristiano más radical en todo lo que la comunidad se tropieza mientras vive. Una de esas cosas es el poder en sus manifestaciones concretas y opresoras ${ }^{40}$. $Y$, aun sobre ello, escribe basado en unas circunstancias determinadas que atraviesa una determinada comunidead.

36. FRIEDRICH-POEHLMANN-STUHLMACHER, a. c., 163.

37. Id., 164. E. KAESEMANN, An die Römer, 346-347. Ensayos exegéticos, 47. U. WIĹCKENS, o. c:; 219. H. SCHLIER, o. c., 391.

38. E. KAESEMANN, Ensayos exegéticos, 47.

39. G. BORNKAMM, o. c., 295.

40. E. KAESEMANN, Ensayos exegéticos, 34. 
Por tanto, es errónea cualquier tentativa de actualización del texto según una idea política: "Nuestro análisis ha mostrado de nuevo que los textos neotestamentarios no deben ser sencillamente actualizados de una forma directa e irreflexiva" ${ }^{41}$. "No estamos en el derecho ni de fundar sobre ellas (las directivas paulinas) un conservadurismo político sin reserva, ni de exigir que respondan a nuestras cuestiones, que Pablo no conoce en su forma actual» ${ }^{42}$.

Wilckens hace un buen estudio de la trayectoria de la interpretación del fragmento, $y$, al final, trata de solucionar el problema que se presenta al cristiano en una sociedad democrática ${ }^{43}$. Se pregunta también por la licitud 0 incluso la obligación de participar en una revolución. En conclusión, hay que decir que el cristiano debe participar normalmente en la vida política, según las posibilidades que su época ofrece $-y$ no quiero canonizar con esto un conformismo de principio: época no significa régimen - , no con desdén, sino precisamente porque le importa la vida de sus contemporáneos. Es evidente que este interés sincero puede llevar a unos a votar a un partido, a otros a votar a otro partido, y a otros a no votar. Pero lo importante es que haya una unión más profunda que la del juego político, por lo que éste queda esencialmente relativizado, desde el momento en que se es cristiano.

En cuanto a la participación en una revolución, también es posible, pero con la misma relativización: "También es claro que no puede haber una revolución cristiana en si misma» ${ }^{44}$, porque ninguna situación coyuntural puede ser el horizonte de un hombre. Este problema nos sitúa muy cerca de aquel otro de la violencia que suele acompañar a toda revolución. Me parece muy acertada la posición de $\mathrm{H}$. Küng a este respecto: «...en un caso límite se puede justificar la violencia - suponiendo que tal justificación tenga sentidocomo legítima defensa del individuo, de un grupo o de una nación. Pero quien recurre a la violencia debe tener muy presente que para ello no puede invocar a Jesús de Nazaret... cuando ha sido 'preciso' derramar sangre, aunque sea sangre realmente culpable, habrá más razones para pedir perdón que para celebrar la revolución” ${ }^{45}$.

Podemos terminar con la frase programática de Käsemann, que pone en su sitio toda resistencia y toda sumisión: «la obediencia cristiana se detiene siempre y solamente en donde ya no es posible el servicio" ${ }^{46}$.

\section{Ángel RODRIGUEZ MERINO}

41 FRIEDRICH-POEHLMANN-STUHLMACHER, a. c., 166.

42. G. BORNKAMM, o. c., 293. E. KAESEMANN, Ensayos exegéticos, 39.

43. U. WILCKENS, o. c., 230-244.

44. E. KAESEMANN, Ensayos exegéticos, 49.

45. H. KUENG, Ser cristiano, Madrid 1977, 720-721.

46. E. KAESEMANN, Ensayos exegéticos, 48. 